\title{
INTERIOR BLOWUP IN A CONVECTION-DIFFUSION EQUATION
}

\author{
CHRISTOPHER P. GRANT*
}

\begin{abstract}
This paper addresses the qualitative behavior of a nonlinear convection-diffusion equation on a smooth bounded domain in $\mathbf{R}^{n}$, in which the strength of the convection grows superlinearly as the density increases. While the initial-boundary value problem is guaranteed to have a local-in-time solution for smooth initial data, it is possible for this solution to be extinguished in finite time. We demonstrate that the way this may occur is through finite-time "blow up", i.e., the unboundedness of the solution in arbitrarily small neighborhoods of one or more points in the closure of the spatial domain. In special circumstances, such as the presence of radial symmetry, the set of blowup points can be identified; these points may be either on the boundary or the interior of the domain. Furthermore, criteria can be established that guarantee that blowup occurs. In this paper, such criteria are presented, involving the dimension of the space, the growth rate of the nonlinearity, the strength of the imposed convection field, the diameter of the domain, and the mass of the initial data. Furthermore, the temporal rate of blowup is estimated.
\end{abstract}

Key words. blowup, convection-diffusion equations

AMS subject classifications. 35B30, 35B40, 35K20, 35K60

1. Introduction. Let $\Omega \subset \mathbf{R}^{n}$ be a bounded domain with smooth boundary, and consider the initial-boundary value problem

$$
\left\{\begin{array}{cc}
u_{t}=\operatorname{div}\left(\nabla u-u^{q} \vec{a}\right) & (x, t) \in \Omega \times(0, T) \\
u_{\nu}=u^{q} \vec{a} \cdot \vec{\nu} & (x, t) \in \partial \Omega \times(0, T), \\
u(x, 0)=u_{0}(x) & x \in \Omega
\end{array},\right.
$$

where $\vec{\nu}$ is the unit outward normal on $\partial \Omega$ and $u_{\nu}$ is the corresponding directional derivative, and (for simplicity) the initial data $u_{0}$ is assumed to be extendable to a smooth, positive function on $\bar{\Omega}$ that satisfies the boundary conditions.

The unknown quantity $u=u(x, t)$ represents the local density of some material that is evolving in response to diffusion and the effects of an imposed convection field $\vec{a}: \bar{\Omega} \rightarrow \mathbf{R}^{n}$. The exponent $q$ (which we shall call the exponent of the nonlinearity) is assumed to be greater than 1 , so the magnitude of the convection term is superlinear in $u$.

Although (1.1) is similar in many respects to equations appearing in the scientific literature (see, e.g., [6] and [10]), the motivation for studying it has less to do with its applicability to any specific physical problem than with its role as an archetype for interacting diffusion and nonlinear convection. In this sense, it plays a role similar to the one that the nonlinear heat equation of Fujita [5]

$$
u_{t}=\Delta u+u^{p}
$$

has come to play for the study of the interaction between diffusion and nonlinear reaction. (For a survey of (1.2) and related equations, see [8].)

The Cauchy problem corresponding to (1.1) in the special case that the convection field is constant has been studied in a series of papers [3], [4], [12]. In that context, solutions decay to zero as $t \rightarrow \infty$, with the asymptotic profile of the decaying solution depending on the particular value of the exponent $q$.

*Department of Mathematics, Brigham Young University, Provo, Utah 84602 USA. 
The one-dimensional version of (1.1) with constant convection

$$
\left\{\begin{array}{cc}
u_{t}=\left(u_{x}+u^{q}\right)_{x} & (x, t) \in(0,1) \times(0, T) \\
u_{x}+u^{q}=0 & (x, t) \in\{0,1\} \times(0, T) \\
u(x, 0)=u_{0}(x) & x \in(0,1)
\end{array}\right.
$$

was studied in [1] and [2]. While solutions to (1.3) stabilize as $t \rightarrow \infty$ if $q<2$, if $q>2$ the convection is strong enough that its concentrating force, in combination with the obstacle posed by the boundary, can overwhelm the diffusion's homogenizing force on the boundary, and solutions can blow up in finite time. By this is meant that there is a time $T$ such that (1.3) has a classical solution for $t \in(0, T)$, but

$$
\limsup _{t \rightarrow T}\|u(\cdot, t)\|_{\infty}=+\infty .
$$

(Here and throughout this paper $\|\psi\|_{p}$ will represent the $L^{p}$ norm on the domain of $\psi$.) The value of $T$ for which (1.4) holds, if it exists, is called the blowup time of the solution.

The purpose of this paper is to address questions about the qualitative behavior of solutions to (1.1): Do solutions exist globally (i.e., for $T=+\infty$ )? Can solutions blow up in finite time? At what points in $\bar{\Omega}$ does blowup occur? What can be said about the asymptotic form of $u$ as the blowup time is approached?

Throughout the remainder of this paper, we will confine our attention to the case that $\vec{a}$ is conservative. More precisely, we shall assume the existence of a continuous function $f: \bar{\Omega} \rightarrow \mathbf{R}$ such that $\nabla f=\vec{a}$. Note that this case includes the case of constant convection.

The first thing that we will show is that the only way solutions to (1.1) can cease to exist is by blowing up:

THEOREM 1.1. Given $u_{0}$ and sufficiently smooth $\vec{a}$, (1.1) has a (unique) positive classical solution for some $T>0$. Furthermore, if $T^{*}$ is the supremum of all $T>0$ for which (1.1) has a solution and $T^{*}<+\infty$, then $\lim \sup _{t \rightarrow T^{*}}\|u(\cdot, t)\|_{\infty}=+\infty$.

In order to obtain more detailed information about the way that blowup can occur, we will focus on the special case of radial symmetry. Consider the following conditions:

(R1) $\Omega=B(0, R):=\left\{x \in \mathbf{R}^{n}:|x|<R\right\}$.

(R2) $\vec{a}(x)=-g(r) x / r$ for some function $g:[0, R] \rightarrow[0, \infty)$. (Here and throughout this paper, $r:=|x|$.)

(R3) $\vec{a}(x)=g(r) x / r$ for some function $g:[0, R] \rightarrow[0, \infty)$.

(R4) $u_{0}(x)=U_{0}(r)$.

The following theorem limits the set of possible blowup points for (1.1). (We will call a point $x \in \bar{\Omega}$ a blowup point if there is not a neighborhood $N$ of $x$ in $\bar{\Omega}$ such that $u$ remains bounded on $N \cap \Omega$.)

THEOREM 1.2. Under conditions $(\mathbf{R 1}),(\mathbf{R 2})$, and (R4), the only possible blowup point for (1.1) is the origin. Under conditions $(\mathbf{R} 1),(\mathbf{R} 3)$, and $(\mathbf{R 4})$, all blowup points lie on the sphere $\partial \Omega$. by

In keeping with our interpretation of $u$ as a density, we define the mass $M$ of $u$

$$
M=M(u)=\int_{\Omega} u d x
$$


Note that (1.1) conserves mass, since

$$
\begin{aligned}
M_{t} & =\left(\int_{\Omega} u d x\right)_{t}=\int_{\Omega} u_{t} d x=\int_{\Omega} \operatorname{div}\left(\nabla u-u^{q} \vec{a}\right) d x \\
& =\int_{\partial \Omega}\left(\nabla u-u^{q} \vec{a}\right) \cdot \vec{\nu} d \sigma=0 .
\end{aligned}
$$

If, in addition to assuming that the convection is unidirectional and radiallyoriented, we assume that the convection is inward at the origin and not too weak, and that the mass is not too small, we can show that finite-time blowup does occur. More precisely, we have the following result:

TheOREM 1.3. Suppose $q>2,(\mathbf{R} 1)$ and $(\mathbf{R 2})$ hold, and there exist constants $C>0$ and $p<n(q-1)-1$ such that $g(r) \geq C r^{p}$ for all $r \in[0, R]$. Then there exists a constant $M_{0}=M_{0}(C, p, q, n, R)$ such that if the mass $M\left(u_{0}\right)>M_{0}$ then the solution $u$ of (1.1) blows up in finite time.

Note that Theorem 1.3 makes no assumption about the radial symmetry of the initial data (and, therefore, of the solution). If this assumption is added then Theorem 1.2 identifies the point where the blowup takes place.

In situations where finite-time blowup does occur and the strength of the convection field can be appropriately bounded, the rate at which blowup occurs can be estimated.

THEOREM 1.4. Suppose hypotheses (R1) and (R2) hold and the solution $u$ of (1.1) blows up at the origin at time $T \in(0, \infty)$. If $g(r) \leq C r^{p}$ for some constants $C>0$ and $p<n(q-1)-1$ and all $r \in[0, R]$ then there exists a constant $K$ such that, for all $t<T$

$$
\|u(\cdot, t)\|_{\infty} \geq \frac{K}{(T-t)^{\beta}}
$$

where

$$
\beta=\frac{p+1}{(n+p+1)(q-1)} .
$$

If, in addition, hypothesis (R4) holds, then (1.5) holds for

$$
\beta=\frac{p+1}{n(q-1)-(p+1)} .
$$

We will proceed as follows. In $\S 2$ we will prove that a unique solution of (1.1) exists locally for smooth initial data and that the only way this solution can fail to be global is for it to blow up. In $\S 3$ we will prove the specific results about radially symmetric systems.

2. Existence until Blowup. For the proof of Theorem 1.1, as well as for other reasons, it will be helpful to understand the set $\mathcal{E}$ of positive equilibrium solutions, i.e. functions $u_{0}: \bar{\Omega} \rightarrow(0, \infty)$ for which $u(x, t) \equiv u_{0}(x)$ solves $(1.1)$.

Suppose $u=u(x)$ is a positive equilibrium solution, and let $w=u^{(1-q)} /(1-q)-f$. A calculation shows that

$$
\operatorname{div}\left(u^{q} \nabla w\right)=0
$$


in $\Omega$ and

$$
u^{q} \frac{\partial w}{\partial \nu}=0
$$

on $\partial \Omega$. Multiplying (2.1) by $w$ and using the generalized divergence theorem and the boundary condition (2.2) yields

$$
\int_{\Omega} u^{q}|\nabla w|^{2} d x=0 .
$$

Thus, $\nabla w \equiv 0$, which implies that

$$
u(x)=\frac{1}{[(q-1)(C-f(x))]^{1 /(q-1)}}
$$

for some constant $C$. It is easy to check that (2.3) defines an equilibrium solution of (1.1) for every $C>\|f\|_{\infty}$.

Among the facts about $\mathcal{E}$ that follow immediately from this explicit formula is the one that we state without proof in the following lemma.

LEMma 2.1. If $\mathcal{E}$ is the set of positive equilibrium solutions of (1.1) and $k \in$ $(0, \infty)$, then

$$
\#\left\{u \in \mathcal{E}:\|u\|_{\infty}=k\right\}=1 .
$$

We now proceed to prove that the only way that solutions can fail to be global is for them to blow up in finite time.

Proof of Theorem 1.1. Let positive initial data $u_{0}$ be given. Pick $b, c>0$ such that $u_{0}(x) \in(b, c)$ for every $x \in \bar{\Omega}$, and pick $b^{\prime} \in(0, b)$ and $c^{\prime} \in(c, \infty)$. Now, choose $h: \mathbf{R} \rightarrow \mathbf{R}$ to be a $C^{\infty}$ function such that $h(\sigma)=\sigma^{q}$ for $\sigma \in\left(b^{\prime}, c^{\prime}\right)$ and $h(\sigma)=0$ if $\sigma<b^{\prime} / 2$ or if $\sigma>2 c^{\prime}$. Let (1.1') be the initial-boundary value problem that results when $u^{q}$ in (1.1) is replaced by $h(u)$. From Theorem 7.4 in [7], we know (1.1') has a unique solution $\tilde{u}$ and it exists globally. (In fact, for given $\theta>0$ existence of a solution in $C^{2+\theta, 1+\theta / 2}$ is guaranteed if $\vec{a} \in C^{1+\theta}$ and $\partial \Omega \in C^{2+\theta}$.) Note that for some $T>0, u=\tilde{u}$ is also a solution of (1.1) for $t<T$. This proves the first half of the theorem.

Let $T^{*}$ be as defined in the statement of the theorem, and suppose

$$
\limsup _{t \rightarrow T^{*}}\|u(\cdot, t)\|_{\infty}<+\infty .
$$

Let $b$ and $c$ be as above. By Lemma 2.1, we can choose $u_{1} \in \mathcal{E}$ with $\left\|u_{1}\right\|_{\infty}<b$. Pick $b^{\prime}>0$ small enough that $u_{1}(x)>b^{\prime}$ for every $x \in \bar{\Omega}$. Pick the value $c^{\prime}>c$ large enough that $u(x, t)<c^{\prime}-1$ for all $t<T^{*}$. Now, consider the solution $\tilde{u}$ to (1.1'). Note that $u_{1}$ is an equilibrium solution for the modified equation, as well, so by the strong maximum principle $\tilde{u}>u_{1}$. Thus, $\tilde{u}$ never leaves the interval $\left(b^{\prime}, c^{\prime}\right)$ where the cutoff function $h$ agrees with $u \mapsto u^{q}$. Hence, $u=\tilde{u}$ is a global solution to (1.1), which implies that $T^{*}=+\infty$. This proves the second half of the theorem.

Throughout the remainder of this paper we shall assume that $\vec{a} \in C^{1+\theta}$ for some $\theta>0$. 
3. Radially Symmetric Systems. The proof of Theorem 1.2 hinges on the existence of quantities which remain bounded and which tie $u$ to $u_{r}$. Such quantities are described in the following lemma.

Lemma 3.1. Suppose hypotheses $(\mathbf{R 1})$ and (R4) are satisfied. Suppose also that initial data $u_{0}$ is given and $u$ is the corresponding solution to (1.1). Then

1. If hypothesis ( $\mathbf{R 2})$ is satisfied then $u$ is radially symmetric and the quantity $Q(x, t):=r^{n-1}\left(u_{r}+u^{q} g(r)\right)$ is bounded on $\Omega \times(0, T)$.

2. If hypothesis (R3) is satisfied then $u$ is radially symmetric and the quantity $Q(x, t):=r^{n-1}\left(u_{r}-u^{q} g(r)\right)$ is bounded on $\Omega \times(0, T)$.

Proof. The radial symmetry of $u$ in either case is a consequence of the uniqueness of solutions to (1.1) (which was established in Theorem 1.1).

Suppose hypotheses (R1), (R2), and (R4) are satisfied, and let

$$
Q(x, t)=r^{n-1}\left(u_{r}+u^{q} g(r)\right) .
$$

A calculation shows that $Q$ satisfies the initial-boundary problem

$$
\left\{\begin{array}{ccc}
Q_{t}=\Delta Q+q u^{q-1} g(r) Q_{r} & & (x, t) \in \Omega \times(0, T) \\
Q=0 & (x, t) \in \partial \Omega \times(0, T) \\
Q(x, 0)=Q_{0}(x, t):=r^{n-1}\left(u_{0 r}+u_{0}^{q} g(r)\right) & x \in \Omega
\end{array}\right.
$$

By the weak maximum principle for parabolic equations (see [9]),

$$
-\left\|Q_{0}\right\|_{\infty} \leq Q(x, t) \leq\left\|Q_{0}\right\|_{\infty}
$$

for all $x \in \Omega$ and all $t>0$. This completes the proof of Part 1 .

Now, suppose hypotheses (R1), (R3), and (R4) are satisfied, and let $Q(x, t)=$ $r^{n-1}\left(u_{r}-u^{q} g(r)\right)$. By an argument similar to the one before, we find, once again, that (3.1) holds for all $x \in \Omega$ and all $t>0$, where, now, $Q_{0}(x)=r^{n-1}\left(u_{0 r}-u_{0}^{q} g(r)\right.$. This completes the proof of Part 2 .

Proof of Theorem 1.2. Under hypotheses (R1), (R2), and (R4), Part 1 of Lemma 3.1 states that the quantity $Q:=r^{n-1}\left(u_{r}+u^{q} g(r)\right)$ is bounded, say by $C$. Thus,

$$
u_{r} \leq \frac{C}{r^{n-1}}-u^{q} g(r)
$$

for all nonzero $x \in \Omega$ and all $t>0$.

If $u$ had a blowup point $x_{0} \neq 0$, then (3.2) would imply the existence of $\varepsilon>0$ and a sequence of times $t_{1}, t_{2}, t_{3}, \ldots$ such that $u\left(x, t_{n}\right)>n$ for every $x \in B\left(0,\left|x_{0}\right|\right) \backslash$ $B\left(0,\left|x_{0}\right|-\varepsilon\right)$. Because of the nonnegativity of $u$, this would contradict the conservation of mass. Hence, 0 is the only possible blowup point.

If hypothesis (R2) is replaced by (R3), Part 2 of Lemma 3.1 states that the quantity $Q:=r^{n-1}\left(u_{r}-u^{q} g(r)\right)$, say by $C$. Thus,

$$
-\frac{C}{r^{n-1}}+u^{q} g(r) \leq u_{r}
$$

for all nonzero $x \in \Omega$ and all $t>0$.

If $u$ had a blowup point $x_{0}$ with $0<\left|x_{0}\right|<R$, then (3.2) would imply the existence of $\varepsilon>0$ and a sequence of times $t_{1}, t_{2}, t_{3}, \ldots$ such that $u\left(x, t_{n}\right)>n$ for every $x \in B\left(0,\left|x_{0}\right|+\varepsilon\right) \backslash B\left(0,\left|x_{0}\right|\right)$. Because of the nonnegativity of $u$, this would again contradict the conservation of mass. 
Now, note that from the definition of blowup point, if $x_{0} \in \bar{\Omega}$ is not a blowup point, then there exists $\delta>0$ such that if $x_{1} \in \bar{\Omega}$ and $\left|x_{1}-x_{0}\right|<\delta$ then $x_{1}$ is not a blowup point either. Hence, the complement of the set of blowup points is open (relative to $\bar{\Omega}$ ) and the set of blowup points is closed (relative to $\bar{\Omega}$ ). In combination with the result of the previous paragraph, this implies that 0 is not a blowup point. Thus, any blowup points must lie on $\partial \Omega$.

The crucial idea in proving Theorem 1.3 is focusing on the amount of mass that has accumulated in a neighborhood of a potential blowup point rather than focusing on the density itself.

Proof of Theorem 1.3. Suppose hypotheses (R1) and (R2) hold and that $p, q$, and $C$ satisfy the conditions in the statment of the theorem. To prove blowup, we measure the concentration of mass near the origin with the variable $w:\left[0, R^{n}\right] \times(0, T)$ defined by

$$
w(\rho, t)=\int_{B\left(0, \rho^{1 / n}\right)} u(x, t) d x .
$$

Straightforward computations reveal that

$$
\begin{gathered}
w_{t}=\int_{S\left(0, \rho^{1 / n}\right)}\left(u_{r}+u^{q} g\left(\rho^{1 / n}\right)\right) d \sigma(x), \\
w_{\rho}=\frac{\rho^{(1-n) / n}}{n} \int_{S\left(0, \rho^{1 / n}\right)} u d \sigma(x)
\end{gathered}
$$

and

$$
w_{\rho \rho}=\frac{\rho^{(2-2 n) / n}}{n^{2}} \int_{S\left(0, \rho^{1 / n}\right)} u_{r} d \sigma(x)
$$

Since $\xi \mapsto \xi^{q}$ is convex on $(0, \infty)$, applying Jensen's Inequality [11] to (3.5) (after scaling $d \sigma(x)$ so that it is a probability measure) and combining it with (3.4) and (3.6) yields

$$
w_{t} \geq n^{2} \rho^{2 \gamma} w_{\rho \rho}+n^{q} \omega_{n}^{1-q} \rho^{\gamma} g\left(\rho^{1 / n}\right) w_{\rho}^{q}
$$

for all $\rho \in\left(0, R^{n}\right)$, where $\omega_{n}$ is the area of the unit sphere in $\mathbf{R}^{n}$ and $\gamma=(n-1) / n$.

Let $p^{\prime}=\max \{p, n-1\}$. Since, by hypothesis, $g(r) \geq C r^{p}$, we have

$$
g(r) \geq C^{\prime} r^{p^{\prime}}
$$

for all $r \in[0, R]$, where $C^{\prime}=C R^{p-p^{\prime}}>0$. Using (3.8) and the fact that, by definition, $w_{\rho}$ is nonnegative, (3.7) implies that

$$
w_{t} \geq n^{2} \rho^{2 \gamma} w_{\rho \rho}+C^{\prime} n^{q} \omega_{n}^{1-q} \rho^{\gamma+p^{\prime} / n} w_{\rho}^{q} .
$$

Now, define

$$
M_{0}=\omega_{n}\left(\frac{p^{\prime}+1}{C^{\prime}(q-1)}\right)^{1 /(q-1)} \frac{R^{n-\left(p^{\prime}+1\right) /(q-1)}}{n-\frac{p^{\prime}+1}{q-1}} .
$$


By hypothesis, $p<n(q-1)-1$ and $q>2$. The first implies that $n-(p+1) /(q-1)>0$ and the second implies that $n-((n-1)+1) /(q-1)>0$. Since either $p^{\prime}=p$ or $p^{\prime}=n-1$, we can conclude that $n-\left(p^{\prime}+1\right) /(q-1)>0$, so $M_{0}$ is well-defined, positive, and finite.

Suppose that $M=M\left(u_{0}\right)>M_{0}$, and set

$$
z(\rho, t)=\left\{\begin{array}{ll}
0 & \text { if } \rho \in[0, \alpha(t)) \\
M\left(\frac{\rho-\alpha(t)}{R^{n}}\right)^{\lambda} & \text { if } \rho \in\left[\alpha(t), R^{n}\right]
\end{array},\right.
$$

where

$$
\lambda=1-\frac{p^{\prime}+1}{n(q-1)}
$$

and $\alpha(t)$ is a continuous, decreasing function of $t$, yet to be determined. Note that, from the discussion in the previous paragraph, $\lambda \in(0,1)$. Thus, in particular, $z$ is continuous.

If possible, we want to choose $\alpha$ so that $z$ can serve as a comparison function with which we can estimate $w$ and, thereby, $u$. In particular, we want $z$ to satisfy the opposite inequality to $(3.9)$; i.e., we want $z$ to satisfy

$$
z_{t} \leq n^{2} \rho^{2 \gamma} z_{\rho \rho}+C^{\prime} n^{q} \omega_{n}^{1-q} \rho^{\gamma+p^{\prime} / n} z_{\rho}^{q}
$$

whenever $\rho \neq \alpha(t)$. Clearly, (3.10) is satisfied whenever $\rho<\alpha(t)$. A straightforward calculation reveals that (3.10) is satisfied for $\rho>\alpha(t)$ if and only if

$$
-\alpha^{\prime}(t) \leq(\rho-\alpha(t))^{-\mu} \rho^{2 \gamma}\left(A \rho^{\mu-1}-B(\rho-\alpha(t))^{\mu-1}\right),
$$

where $\mu=\left(p^{\prime}+1\right) / n$,

$$
A=C^{\prime}\left(\frac{M \lambda}{\omega_{n}}\right)^{q-1} n^{q} R^{n(\mu-q+1)},
$$

and $B=n^{2}(1-\lambda)$. Another calculation reveals that, because $M>M_{0}, A>B$. Since $\rho>\alpha(t), \rho>\rho-\alpha(t)$, and $\mu-1 \geq 0$, this means that (3.11) will be satisfied if

$$
-\alpha^{\prime}(t) \leq(A-B)(\alpha(t))^{1-2 / n} .
$$

If we take $\alpha(t)$ to be the solution of the initial value problem

$$
\left\{\begin{array}{c}
\alpha^{\prime}(t)=-(A-B)(\alpha(t))^{1-2 / n} \\
\alpha(0)=R^{n}
\end{array}\right.
$$

then (3.12) will be satisfied and, furthermore, there will be some finite $T^{*}$ such that $\alpha(t) \rightarrow 0$ as $t \rightarrow T^{*}$.

Now, suppose that the solution $u$ to (1.1) does not blow up by time $T^{*}$. Then, by Theorem 1.1, $u$ is defined for $t \in\left[0, T^{*}\right]$. Consider the function $y:\left[0, R^{n}\right] \times\left[0, T^{*}\right] \rightarrow \mathbf{R}$ defined by $y(\rho, t)=e^{-t}(z(\rho, t)-w(\rho, t))$. We claim that $y \leq 0$ on its domain $D$. If it is not, then since $y$ is continuous, it must achieve a positive maximum on $D$.

Note that

$$
y(\rho, 0)=z(\rho, 0)-w(\rho, 0)=-\int_{B\left(0, \rho^{1 / n}\right)} u_{0}(x) d x \leq 0,
$$


$y(0, t)=e^{-t}(z(0, t)-w(0, t))=0$, and

$$
y\left(R^{n}, t\right)=e^{-t}\left(z\left(R^{n}, t\right)-w\left(R^{n}, t\right)\right)=e^{-t}\left(M\left(\frac{R^{n}-\alpha(t)}{R^{n}}\right)^{\lambda}-M\right) \leq 0,
$$

so $y$ does not achieve a positive maximum on

$$
\left\{(\rho, 0): \rho \in\left[0, R^{n}\right]\right\} \cup\left\{(0, t): t \in\left[0, T^{*}\right]\right\} \cup\left\{\left(R^{n}, t\right): t \in\left[0, T^{*}\right]\right\} .
$$

Also, because $\lambda<1$,

$$
\lim _{\rho \rightarrow \alpha(t)^{+}} z_{\rho}(\rho, t)=-\infty
$$

so $y$ cannot achieve a positive maximum at a point where $\rho=\alpha(t)$. This implies that at a positive maximum, $y$ must satisfy $y_{\rho}=0, y_{\rho \rho} \leq 0$, and $y_{t} \geq 0$. But, using (3.9) and (3.10), that would mean that at such a point

$$
\begin{aligned}
y_{t} & =-y+e^{-t}\left(z_{t}-w_{t}\right) \\
& \leq-y+e^{-t}\left(n^{2} \rho^{2 \gamma}(z-w)_{\rho \rho}+C^{\prime} n^{q} \omega_{n}^{1-q} \rho^{\gamma+p^{\prime} / n}\left(z_{\rho}^{q}-w_{\rho}^{q}\right)\right) \\
& =-y+n^{2} \rho^{2 \gamma} y_{\rho \rho}<0,
\end{aligned}
$$

which is a contradiction.

This contradiction verifies the nonnegativity of $y$, which means that $w \geq z$ on all of $D$. But it is not hard to see that $\sup \left\{z(\rho, t) / \rho: \rho \in\left(0, R^{n}\right)\right\}$ becomes unbounded as $t \rightarrow T^{*}$, so $\sup \left\{w(\rho, t) / \rho: \rho \in\left(0, R^{n}\right)\right\}$ must also become unbounded. From the definition of $w$ and the Mean Value Theorem for integrals, this, in turn, implies that $\|u(\cdot, t)\|_{\infty}$ becomes unbounded as $t \rightarrow T^{*}$, contradicting the assumption that $u$ does not blow up by time $T^{*}$. This completes the proof.

In order to prove the estimates on the temporal blowup rate, it will be helpful to have the following technical lemma, which estimates the degree to which equilibrium solutions concentrate mass near the origin.

LEMMA 3.2. Suppose that (R1) and (R2) hold and that $g(r) \leq C r^{p}$ for some $p<n(q-1)-1$ and all $r \in[0, R]$, and let

$$
w_{c}(\rho)=\int_{B\left(0, \rho^{1 / n}\right)} u_{c}(x) d x,
$$

where

$$
u_{c}(x)=\sup \{u(x): u \in \mathcal{E}\} .
$$

Then

$$
\rho \mapsto-\int_{B\left(0, \rho^{1 / n}\right)} u(x) d x
$$

is convex for any $u \in \mathcal{E}$, and so is $-w_{c}$. Furthermore, there exists a positive constant $K^{\prime}$ such that

$$
\sup \left\{w_{c}(\rho)-\lambda \rho: \rho \in\left[0, R^{n}\right]\right\} \geq K^{\prime} \lambda^{\mu}
$$


for every $\lambda$ sufficiently large, where

$$
\mu=1-\frac{n(q-1)}{p+1} .
$$

Proof. It is not hard see (e.g., by examining (2.3)) that $u_{c}$ is radially symmetric and satisfies the same equation as the members of $\mathcal{E}$ but has a singularity at the origin. Furthermore, its radial symmetry implies that equality holds in (3.7) for $w=w_{c}$ (because Jensen's Inequality is an equality in that case). Hence,

$$
w_{c}^{\prime \prime}=-K^{\prime} \rho^{-(n-1) / n} g\left(\rho^{1 / n}\right)\left(w_{c}^{\prime}\right)^{q}
$$

for some positive constant $K^{\prime}$. (Throughout this proof, $K^{\prime}$ will represent a positive constant whose value may change from line to line.) Note that (3.15) implies the convexity of $-w_{c}$, and since

$$
\rho \mapsto-\int_{B\left(0, \rho^{1 / n}\right)} u(x) d x
$$

satisfies a similar equation for any $u \in \mathcal{E}$, it is convex, also.

Integrating (3.15) and using the fact that $w_{c}^{\prime}(\rho) \rightarrow+\infty$ as $\rho$ approaches zero from the right, yields

$$
w_{c}^{\prime}(\rho)=K^{\prime}\left(\int_{0}^{\rho} \sigma^{(1-n) / n} g\left(\sigma^{1 / n}\right) d \sigma\right)^{-1 /(q-1)} .
$$

Applying the fact that $w_{c}(0)=0$ and the assumption that $g(r) \leq C r^{p}$ in (3.16) yields

$$
w_{c}(\rho) \geq K^{\prime} \rho^{\mu}
$$

where $\mu$ is as in (3.14). Using (3.17), it is a straightforward calculus exercise to verify that (3.13) holds for all $\lambda$ sufficiently large.

Lemma 3.2 provides a crucial estimate for constructing a type of supersolution that will provide an estimate of the blowup rate.

Proof of Theorem 1.4. Assume that the hypotheses (R1) and (R2) hold and that $g(r) \leq C r^{p}$ for some constants $C>0$ and $p<n(q-1)-1$ and for all $r \in[0, R]$. Assume also that the solution $u$ of (1.1) blows up at the origin at time $T \in(0, \infty)$. Fix $t_{0} \in(0, T)$ and let $\lambda=\left(\omega_{n} / n\right)\left\|u\left(\cdot, t_{0}\right)\right\|_{\infty}$.

Pick $\tilde{u} \in \mathcal{E}$ such that $\|\tilde{u}\|_{\infty}>\left\|u\left(\cdot, t_{0}\right)\right\|_{\infty}$ and let

$$
\tilde{w}(\rho)=\int_{B\left(0, \rho^{1 / n}\right)} \tilde{u}(x) d x .
$$

For $y$ between 0 and

$$
G[\tilde{w}]:=\sup \left\{\tilde{w}(\rho)-\lambda \rho: \rho \in\left[0, R^{n}\right]\right\},
$$

let $\varrho(y)$ be the leftmost zero of $\tilde{w}(\rho)-\lambda \rho-y$. For $t \geq t_{0}$ consider the function

$$
z(\rho, t)=\left\{\begin{array}{ll}
\tilde{w}(\rho) & \text { if } \rho \in\left[0, \varrho\left(v\left(t-t_{0}\right)\right]\right. \\
\lambda \rho+v\left(t-t_{0}\right) & \text { if } \rho \in\left[\varrho\left(v\left(t-t_{0}\right), R^{n}\right]\right.
\end{array},\right.
$$


where $v$ is a positive constant to be specified later. The intention is that by picking $v$ appropriately, $z(\rho, t)$ will serve as an upper bound for

$$
w(\rho, t):=\int_{B\left(0, \rho^{1 / n}\right)} u(x, t) d x,
$$

and, therefore, $\|\tilde{u}\|_{\infty}$ will bound $u$ in a neighborhood of the origin.

Under the assumptions made above, let $v=C R^{p} n^{q} \omega_{n}^{1-q} \lambda^{q}$. We claim that $w \leq z$ as long as $z$ is well-defined (i.e., until $\tilde{w}(\rho)-\lambda \rho-v\left(t-t_{0}\right)$ has no zeros). By the strong maximum principle, it suffices to prove that $W \leq z$, where

$$
W(\rho, t):=\int_{B\left(0, \rho^{1 / n}\right)} U(x, t) d x
$$

and $U$ is a radially symmetric solution of (1.1) for $t \geq t_{0}$ that satisfies $u\left(x, t_{0}\right) \leq$ $U\left(x, t_{0}\right) \leq\left\|u\left(\cdot, t_{0}\right)\right\|_{\infty}$.

Suppose that $W(\rho, t)>z(\rho, t)$ for some $\rho \in\left[0, R^{n}\right]$ and some $t \geq t_{0}$. Note that by the choice of $\lambda, W\left(\rho, t_{0}\right) \leq z\left(\rho, t_{0}\right)$. Also, $W(0, t)=z(0, t)=0$ and $W\left(R^{n}, t\right)=$ $W\left(R^{n}, t_{0}\right) \leq z\left(R^{n}, t_{0}\right) \leq z\left(R^{n}, t\right)$ for every $t \geq t_{0}$. Thus, there must be some $t_{1}>t_{0}$ and $\rho_{1} \in\left(0, R^{n}\right)$ for which $\zeta:=(W-z) e^{-t}$ satisfies $\zeta\left(\rho_{1}, t_{1}\right)>0$ and $\zeta\left(\rho_{1}, t_{1}\right) \geq \zeta(\rho, t)$ for every $t \in\left[t_{0}, t_{1}\right]$ and every $\rho \in\left[0, R^{n}\right]$. Note that $\left(\rho_{1}, t_{1}\right)$ cannot be a point $\left(\varrho\left(v\left(t_{1}-t_{0}\right)\right), t_{1}\right)$ at which $\zeta$ is not smooth because $W$ is continuously differentiable and the limit of $z_{\rho}\left(\rho, t_{1}\right)$ as $\rho$ approaches $\varrho\left(v\left(t_{1}-t_{0}\right)\right)$ from the left is higher than the corresponding righthand limit. Hence, it must be the case that

$$
\begin{aligned}
\zeta_{\rho}\left(\rho_{1}, t_{1}\right) & =0, \\
\zeta_{\rho \rho}\left(\rho_{1}, t_{1}\right) & \leq 0,
\end{aligned}
$$

and

$$
\zeta_{t}\left(\rho_{1}, t_{1}\right) \geq 0
$$

Equation (3.19) implies that

$$
W_{\rho}\left(\rho_{1}, t_{1}\right)=z_{\rho}\left(\rho_{1}, t_{1}\right)
$$

and (3.20) implies that

$$
W_{\rho \rho}\left(\left(\rho_{1}, t_{1}\right) \leq z_{\rho \rho}\left(\rho_{1}, t_{1}\right) .\right.
$$

But, because $z=\tilde{w}$ for $\rho<\varrho\left(v\left(t_{1}-t_{0}\right)\right)$ and because of the choice of $v$,

$$
z_{t} \geq n^{2} \rho^{2 \gamma} z_{\rho \rho}+n^{q} \omega_{n}^{1-q} \rho^{\gamma} g\left(\rho^{1 / n}\right) z_{\rho}^{q}
$$

at $\left(\rho_{1}, t_{1}\right)$. Also, the radial symmetry of $W$ implies that

$$
W_{t}=n^{2} \rho^{2 \gamma} W_{\rho \rho}+n^{q} \omega_{n}^{1-q} \rho^{\gamma} g\left(\rho^{1 / n}\right) W_{\rho}^{q} .
$$

Using equations (3.22) through (3.25) we find that at $\left(\rho_{1}, t_{1}\right)$

$$
\zeta_{t}=-\zeta+\left(W_{t}-z_{t}\right) e^{-t} \leq-\zeta<0,
$$

which contradicts (3.21), so $w(\rho, t) \leq z(\rho, t)$ for all $\rho \in\left[0, R^{n}\right]$ and all $t \geq t_{0}$ for which $z$ is defined. As was mentioned above, this implies that $u$ does not blow up in this time interval. 
Note that the length of this time interval past $t_{0}$ on which $u$ is guaranteed not to blow up is proportional to $G[\tilde{w}]$ (because of the convexity of $-\tilde{w}$ ) and inversely proportional to $v$. Letting $\tilde{u} \rightarrow u_{c}$ (and, therefore, $\tilde{w} \rightarrow w_{c}$ ) and using Lemma 3.2 (which estimates $G\left[w_{c}\right]$ ), we find that

$$
T-t_{0} \geq \tilde{C}\left\|u\left(\cdot, t_{0}\right)\right\|_{\infty}^{\mu-q},
$$

for some constant $\tilde{C}$ (independent of $t_{0}$ ), if $\left\|u\left(\cdot, t_{0}\right)\right\|_{\infty}$ is sufficiently large. It is not hard to see that (by possibly decreasing $\tilde{C}$ ) we can get (3.26) to hold for $t_{0}$ bounded away from $T$, as well, so (3.26) holds for all $t_{0} \geq 0$. Replacing $t_{0}$ by $t$ and rewriting (3.26) we get (1.5) and (1.6).

Now, suppose that the hypothesis of radial symmetry for $u$ is added. Let $w$ be defined as in (3.18). By Part 1 of Lemma 3.1, $Q:=r^{n-1}\left(u_{r}+u^{q} g(r)\right)$ is bounded by a constant $v$, and a calculation shows that $w_{t}=Q$. Using this fact, an improved lower bound on the size of the remaining interval existence until blowup can be obtained in much the same way as the previous bound. In particular, we can define $z$ as previously but pick $v$ in the definition of $z$ to be equal to the constant that bounds $Q$. An argument similar to the one used before implies that $w \leq z$ as long as $z$ is defined; the only difference is that for $\rho>\varrho\left(v\left(t_{1}-t_{0}\right)\right)$, the inequality (3.24) no longer holds so we use the fact that the constant $v$ bounds $w_{t}$ in place of the combination of (3.24) and (3.25) to conclude directly that $z_{t} \geq w_{t}$ for such $\rho$. Since $v$ is now independent of $\lambda$, we obtain

$$
T-t_{0} \geq \tilde{C}\left\|u\left(\cdot, t_{0}\right)\right\|_{\infty}^{\mu}
$$

in place of (3.26). The estimate (1.5) with (1.7) is an immediate consequence.

\section{REFERENCES}

[1] N. D. Alikakos, P. W. Bates, and C. P. Grant, Blow up for a diffusion-advection equation, Proc. Roy. Soc. Edinburgh Sect. A, 113 (1989), pp. 181-190.

[2] G. R. Conner And C. P. Grant, Asymptotics of blowup for a convection-diffusion equation with conservation, Differential Integral Equations, 9 (1996), pp. 719-728.

[3] M. Escobedo, J. L. VÁzquez, and E. Zuazua, A diffusion-convection equation in several space dimensions, Indiana Univ. Math. J., 42 (1993), pp. 1413-1440.

[4] M. Escobedo And E. ZuAzUA, Large time behavior for convection-diffusion equations in $\mathbf{R}^{n}$, J. Funct. Anal., 100 (1991), pp. 119-161.

[5] H. FuJita, On the blowing up of solutions of the cauchy problem for $u_{t}=\delta u^{\sigma+1}+u^{\beta}$, J. Fac. Sci. Univ. Tokyo Sect. 1A Math., 16 (1966), pp. 105-113.

[6] R. E. Grundy, C. J. van Duijn, and C. N. Dawson, Asymptotic profiles with finite mass in one-dimensional contaminant transport through porous media: The fast reaction case, Quart. J. Mech. Appl. Math., 94 (1994), pp. 69-106.

[7] O. A. Ladyzenskaja, V. A. Solonnikov, and N. N. Ural'ceva, Linear and Quasilinear Equations of Parabolic Type, vol. 23 of Translations of Mathematical Monographs, American Mathematical Society, Providence, 1968.

[8] H. A. Levine, The role of critical exponents in blowup theorems, SIAM Rev., 32 (1990), pp. $262-288$.

[9] M. H. Protter and H. F. Weinberger, Maximum Principles in Differential Equations, Prentice-Hall, Englewood Cliffs, NJ, 1967.

[10] P. Rosenau And S. Kamin, Thermal waves in an absorbing and convecting medium, Phys. D, 8 (1983), pp. 273-283.

[11] W. Rudin, Real and Complex Analysis, McGraw-Hill, New York, third ed., 1987.

[12] E. ZuAZuA, Weakly nonlinear large time behavior in scalar convection-diffusion equations, Differential Integral Equations, 6 (1993), pp. 1481-1491. 